\title{
Severe chest pain with mid-ventricular obstruction in a patient with hyperthyroidism
}

\author{
Jong-Ho Nam, Jang Won Son ${ }^{2}$, Geu-Ru Hong ${ }^{1}$ \\ ${ }^{l}$ Cardiology Division, Severance Cardiovascular Hospital, Yonsei University College of Medicine, Seoul; \\ ${ }^{2}$ Division of Cardiology, Department of Internal Medicine, Yeungnam University College of Medicine, Daegu, Korea
}

Mid-ventricular obstruction (MVO) rarely occurs in patients without hypertrophic cardiomyopathy. Increased cardiac contractility may play an important role in causing MVO. We experienced a case of severe chest pain and MVO in a 50-year-old female patient. She had hypertension, diabetes, stroke and peripheral artery disease. Her blood pressure was very high $(222 / 122 \mathrm{mmHg})$ with severe fluctuation. The transthoracic echocardiography revealed MVO accompanied by hyper-dynamic left ventricular systolic function. We regarded her chest pain and MVO as secondary findings related to other diseases. Coronary angiography and several tests for uncontrolled hypertension were performed, and those evaluations revealed that she had coronary artery disease and hyperthyroidism. We considered that the increase in the myocardial oxygen demand in response to the increase in cardiac contractility and workload associated with hyperthyroidism aggravated her symptoms and MVO. She was treated with methimazole and beta blockers and her symptoms dramatically improved.

Keywords: Hyperthyroidism; Coronary artery disease; Ventricular function

\section{INTRODUCTION}

Mid-ventricular obstruction (MVO) occurs in approximately $10 \%$ of patients with hypertrophic cardiomyopathy (HCM) [1]. MVO in patients with HCM is characterized by an impedance to the flow that occurs at the midcavitary level, and is predominantly caused by marked septal hypertrophy coming in contact with a hyper-contractile left ventricular free wall [2]. Even without HCM, MVO can occur in fewer patients with small ventricular chambers or increased cardiac contractility [3]. We describe a case of a woman presenting chest

Received: April 3, 2017, Revised: April 24, 2017

Accepted: May 4, 2017

Corresponding Author: Jang Won Son, Division of Cardiology, Department of Internal Medicine, Yeungnam University College of Medicine, 170 Hyeonchung-ro, Nam-gu, Daegu 42415, Korea

Tel: +82-53-620-3314, Fax: +82-53-621-3310

E-mail: gubjae@yu.ac.kr pain and MVO, which were clues to the identification of proper diagnosis and treatment.

\section{CASE}

A 50-year-old female with a history of hypertension, diabetes, stroke and peripheral artery disease presented to the cardiology department due to aggravated chest pain accompanied by severe blood pressure fluctuations. She had been hospitalized in the thoracic surgery department for an excisional biopsy of a lung mass with suspicion of malignancy. Upon presentation, her arterial pressure was $222 / 122 \mathrm{mmHg}$ and pulse rate was 113 beats per minutes (bpm). On physical examination, an apical systolic murmur (grade 3/6), which was increased to grade 4/6 during Valsalva maneuver, was determined on auscultation and her pulse was bounding. Electrocardiography demonstrated sinus tachycardia with a rate of 103 bpm. Routine laboratory tests including the cardiac troponin

Copyright (C) 2017 Yeungnam University College of Medicine

This is an Open Access article distributed under the terms of the Creative Commons Attribution Non-Commercial License (http://creativecommons.org/licenses/by-nc/4.0/) which permits unrestricted non-commercial use, distribution, and reproduction in any medium, provided the original work is properly cited. 
level were within normal limits. Transthoracic echocardiography (TTE) was performed, revealing a severe dynamic obstruction of the mid-ventricle (maximal pressure gradient of $96 \mathrm{mmHg}$ at rest, and $117 \mathrm{mmHg}$ during Valsalva maneuver) accompanied by mild concentric left ventricular hypertrophy and hyper-dynamic left ventricular systolic function (ejection fraction, 76\% [range, 55-75\%]) (Fig. 1).

Thoracic surgeons focused on MVO, as they thought her symptoms were related to MVO itself; they considered performing a myectomy. However, we considered that her symptoms and MVO could have been secondary findings related to other diseases. First, given her high risk for coronary artery disease (CAD), coronary angiography (CAG) was performed. CAG revealed significant stenosis in the proximal left anterior descending artery, as well as in the proximal and distal segments of the left circumflex artery (Fig. 2). Second, to evaluate her high and severely fluctuating blood pressure, in addition to her increased cardiac contractility with MVO, we performed a thyroid function test, 24-hour urine metanephrine and vanillylmandelic acid excretion levels, serum renin and aldosterone levels, as well as computed tomography of the abdomen to evaluate for secondary hypertension. The tests revealed that she had hyperthyroidism with a decreased thyroid stimulation hormone (TSH, $<0.16 \mathrm{mu} / \mathrm{L}$ [range, $0.3-4 \mathrm{mu} / \mathrm{L}]$ ), increased T3 $(1.32 \mathrm{nmol} / \mathrm{L}$ [range, $1.23-3.08 \mathrm{nmol} / \mathrm{L}$ ]), increased free T4 (84.05 pmol/L [range, 10-25 pmol/L]) levels, and a positive TSH receptor antibody test $(4.48 \mathrm{IU} / \mathrm{dL}$ [range, 0 $1.5 \mathrm{IU} / \mathrm{dL}])$.

She was treated with methimazole (10 mg twice a day), dexamethasone ( $0.5 \mathrm{mg}$ twice a day for 1 week), and beta blockers (carvedilol $25 \mathrm{mg}$ twice a day) and just after 3 days of treatment, her symptoms dramatically improved without cor-
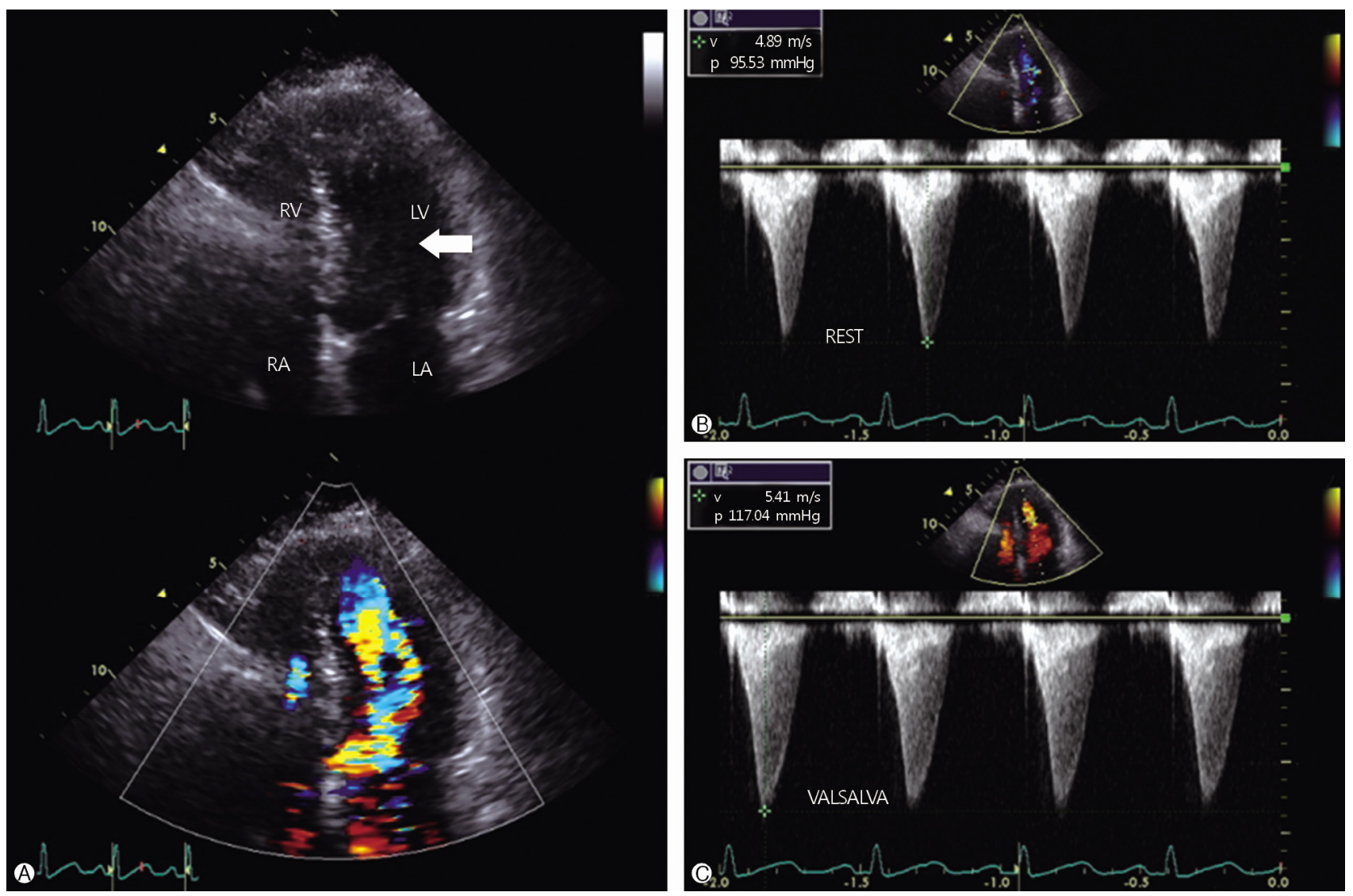

Fig 1. In an apical four-chamber view of the 2 dimensional TTE and color-flow Doppler imaging (A), a MVO (arrow) during the end-systole (top) results in a poststenotic turbulence (bottom). Continuous-wave Doppler imaging (B and C) exhibits high velocity, late-peaking jet typical of a dynamic MVO by a Valsalva maneuver. At rest, Doppler demonstrates a late-peaking systolic jet of $4.89 \mathrm{~m} / \mathrm{s}$ (B). After the Valsalva maneuver, the maximal velocity is $5.41 \mathrm{~m} / \mathrm{s}(\mathrm{C})$. TTE, transthoracic echocardiography; MVO, mid-ventricular obstruction; LA, left atrium; LV, left ventricle; RA, right atrium; RV, right ventricle. 
Jong-Ho Nam et al.
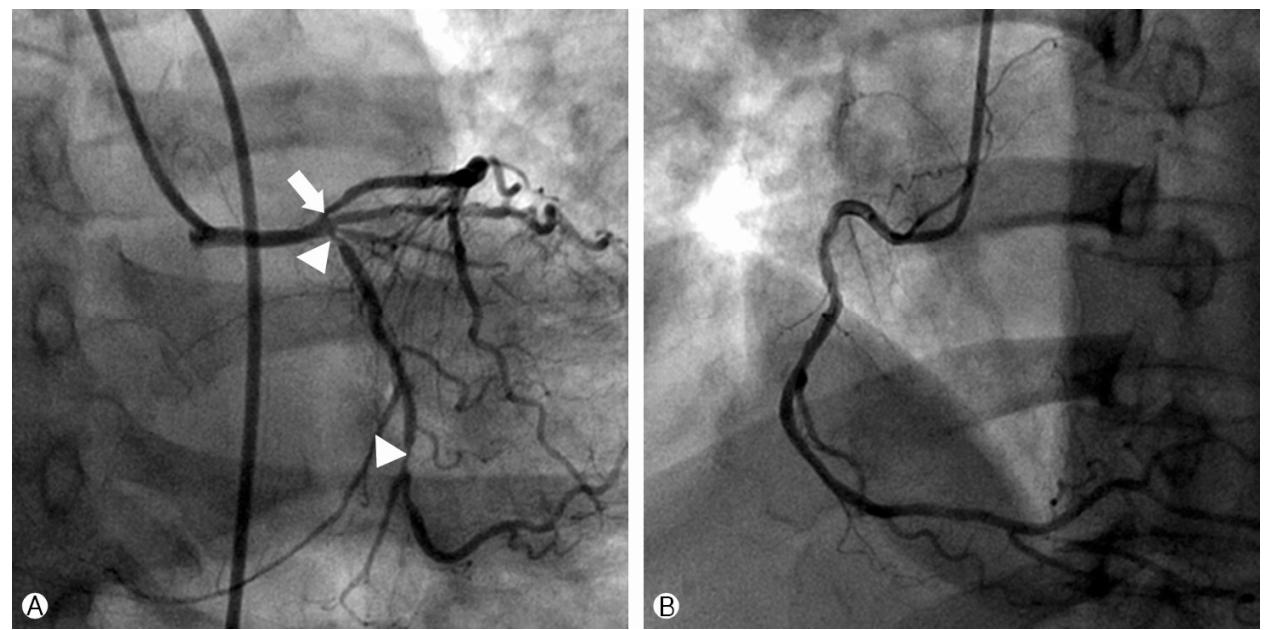

Fig 2. CAG of the left coronary artery (A) shows significant steno- sis in the proximal left anterior descending artery (arrow), as well as in the proximal and distal segments of the left circumflex artery (arrow head). CAG of the right coronary artery (B) reveals no critical stenosis. CAG, coronary angiography.
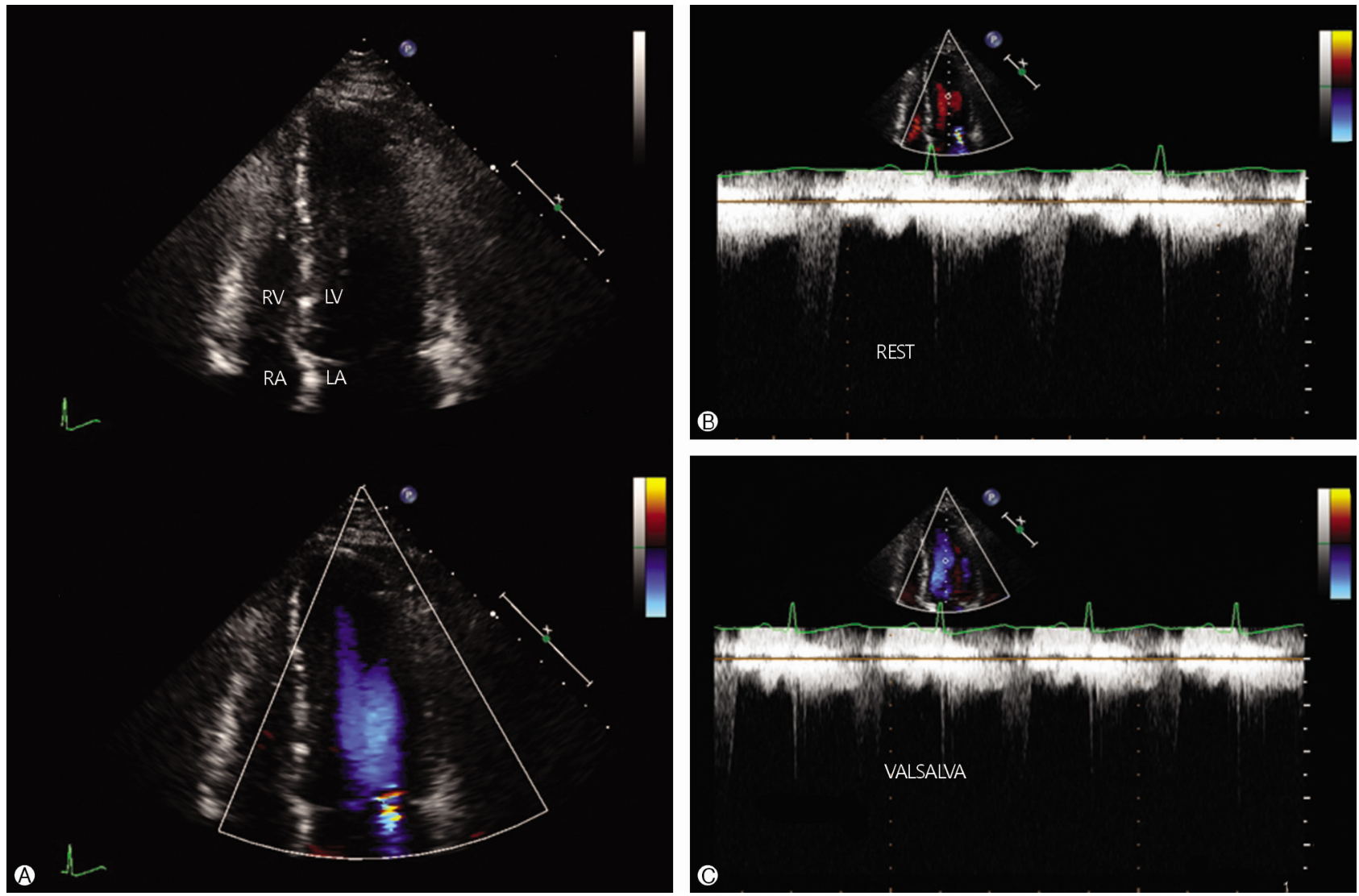

Fig 3. In an apical four-chamber view of the 2 dimensional TTE and color-flow Doppler imaging (A), the MVO during end-systole (top) and poststenotic turbulence disappear (bottom). The high velocity, late-peaking jet typical of a MVO is no longer seen in the continuous-wave Doppler imaging even after the Valsalva maneuver (B and C). TTE, transthoracic echocardiography; MVO, mid-ventricular obstruction; LA, left atrium; LV, left ventricle; RA, right atrium; RV, right ventricle. 
onary revascularization and her blood pressure was controlled at $134 / 66 \mathrm{mmHg}$ with a heart rate of $80 \mathrm{bpm}$. Follow-up TTE revealed a disappearance of MVO and a normalized ejection fraction of $70 \%$ (Fig. 3). After 2 weeks of treatment, she underwent coronary artery bypass surgery and a concomitant lobectomy of a lung mass via a video-assisted thoracoscopic surgery. The lung mass was confirmed as an adenocarcinoma.

\section{DISCUSSION}

We detailed a case of a woman with chest pain and MVO caused by hyperthyroidism. MVO in patients with HCM is defined as peak instantaneous Doppler mid-ventricular pressure gradient of $\geq 30 \mathrm{mmHg}$, but the threshold for invasive treatment is usually considered to be $\geq 50 \mathrm{mmHg}[1,2]$. Before considering invasive treatment, a systematic assessment, such as whether there are any alternative or additional explanations for the symptoms, is essential for symptomatic patients with MVO gradient $\geq 50 \mathrm{mmHg}$, despite maximally tolerated drug therapy [1]. For clinical purposes, it seems reasonable to expand this guideline to include symptomatic MVO in patients without $\mathrm{HCM}$, despite little supporting data due to its relative rarity. We considered that hyperthyroidism was the true cause of her high blood pressure, increased cardiac contractility, and $\mathrm{MVO}$, which led to an increased myocardial oxygen demand and an aggravation of chest pain with underlying CAD [4]. Finally, we were able to relieve her chest pain and reduced the risk of unnecessary myectomy or coronary revascularization by treating the hyperthyroidism itself.
Our case showed that MVO could be a clue to diagnose hidden underlying diseases, such as hyperthyroidism. Careful consideration and evaluation of the underlying cause of MVO can provide an important clue in making a diagnosis and leading to an appropriate treatment.

\section{CONFLICT OF INTEREST}

The authors have no financial conflicts of interest.

\section{ORCID}

Jong-Ho Nam, https://orcid.org/0000-0001-5106-8361

Jang-Won Son, https://orcid.org/0000-0002-8109-5018

\section{REFERENCES}

1. Elliott PM, Anastasakis A, Borger MA, Borggrefe M, Cecchi F, Charron P, et al. 2014 ESC Guidelines on diagnosis and management of hypertrophic cardiomyopathy: the Task Force for the Diagnosis and Management of Hypertrophic Cardiomyopathy of the European Society of Cardiology (ESC). Eur Heart J 2014;35:2733-79.

2. Minami Y, Kajimoto K, Terajima Y, Yashiro B, Okayama D, Haruki S, et al. Clinical implications of midventricular obstruction in patients with hypertrophic cardiomyopathy. J Am Coll Cardiol 2011;57:2346-55.

3. Murakami H, Nishimura M, Urabe K. Relation between dynamic midventricular obstruction and unexplained chest pain in patients with normal echocardiograms at rest. Am J Cardiol 1996;78:1063-5.

4. Klein I, Danzi S. Thyroid disease and the heart. Circulation. 2007;116:1725-35. 